\title{
A Conceptual Model of the Axiomatic Usability Evaluation Method
}

\author{
Yinni Guo ${ }^{1}$, Robert W. Proctor ${ }^{1,2}$, and Gavriel Salvendy ${ }^{1,3}$ \\ ${ }^{1}$ School of Industrial Engineering, Purdue University, West Lafayette, IN \\ ${ }^{2}$ Department of Psychological Sciences, Purdue University, West Lafayette, IN, USA \\ ${ }^{3}$ Department of Industrial Engineering, Tsinghua University, Beijing, China \\ \{guo2, rproctor, salvendy\} @purdue.edu
}

\begin{abstract}
This paper describes a new usability evaluation method developed for consumer electronics. This method, Axiomatic Evaluation, is based on Axiomatic Design theory, a formalized methodology that can be used to represent a variety of design problems. From this perspective, to generate a design that meets the perceived needs, designers should first specify the design goals of "what we want to achieve", and then start the design process with a clear description of "how we will achieve it", so that the recursive "design/build/test" cycle could be reduced.
\end{abstract}

Keywords: axiomatic design, usability engineering.

\section{Introduction}

Usability has been studied in Human-Computer Interaction (HCI) for about two decades to improve the efficiency and effectiveness of interactions. Several evaluation methods have been used to detect usability problems, mostly for websites and software but also for other products. However, some authors have argued that HCI usability methods are not well suited for application to consumer electronic products. Consequently, our objective was to develop and describe a new usability evaluation method for consumer electronic products.

\section{Background Literature}

\subsection{Usability Evaluation Methods}

Usability evaluation seeks the best way to improve the quality of systems or products in terms of effectiveness, efficiency, and satisfaction. Traditional usability evaluation methods, including cognitive walkthrough, heuristic evaluation, think aloud, interview, focus group, and questionnaire, are widely used to test computer-based products like software and web sites, and have met with good success. However, some authors suggest that the same concept of software usability cannot be employed effectively for consumer electronic products. For example, Han, Yun, Kim, and Kwahk stated, "It is not appropriate to apply the same concept of software usability developed in the 
HCI research directly to the consumer electronic products" [1]. One reason for this assessment is that there are several aspects of the interfaces and interactions with consumer electronic devices that are different from those of the computer-based products [2]. For computer-based products like web sites or software, users can use the keyboard or mouse to control the interface. These control tools, especially the mouse, can be moved freely and quickly all over the interface. Compared to these set- ups on the computer, control of the interfaces for consumer electronic products is limited and more difficult.

Compared to computer-based products like software and websites, consumer electronic products like mobile phones impose limitations such as smaller screen size and cumbersome input mechanisms [3]. Also, because the consumer electronic products have hardware-oriented features such as dedicated controls in addition to softwareoriented features such as menus, there are more dimensions that need to be considered with regard to usability [4]. Unlike just clicking the mouse, users of consumer electronic products typically need to press the control panel or remote control several times when they want to change a setting or go to a certain function. Sometimes they cannot even determine how to operate the device because there is not enough information about control actions or the design of operation does not fit the users' mental models [1]. Users may encounter situations of pressing the button more times than needed and then have to spend additional time pressing the keys again. A typical example is how users change their television or monitor settings.

Because of these differences, researchers have tried to establish distinct usability guidelines for consumer electronic products. For mobile devices, guidelines include: five usability criteria for mobile UI design [5], a usability checklist based on 21 usability principles [6], and nine critical factors for handset's perceived usability [2]. Besides the studies of the devices, service for consumer electronic products has also been investigated to enhance the usability. It has been suggested that personalization is essential for creating a positive mobile experience [3,7]. Similar to web-based products, the "fun" attribute contributes to users' adoption of wireless handheld devices, even more than the perceived usefulness does [8].

\subsection{Axiomatic Design}

Introduction. How the design process should be structured is always an issue. In the past, many engineers have designed their products interactively, empirically, and intuitively, based on creative insights from years of experience. Undisciplined designs have resulted that are not only poor in terms of customer satisfaction, but also with respect to missed schedules, cost overruns and failed products. Symptoms of poor design include low quality and unreliability, as well as high costs of hardware, software, and systems [9]. Some design errors are major problems that have been well publicized. However, there are also many "small" problems that simply inconvenience or aggravate consumers. All bad designs can be dangerous, cost money, limit the usefulness of products, or delay the introduction of new products.

To be efficient and to generate a design that meets the perceived needs, the designer must specifically state the design goals before the design process begins. Therefore, a rigorous design approach must start with an explicit statement of "what we want to achieve" and end with a clear description of "how we will achieve it" [10]. 
Once the customers' needs are understood, these needs must be transformed into a minimum set of specifications, or functional requirements, that adequately describe "what we want to achieve" to satisfy the customers' needs. The descriptor of "how to achieve it" may be in the form of design parameters.

Axiomatic design is a formalized methodology that can be used to represent a variety of design problems [9]. The ultimate goal of axiomatic design is to establish a scientific basis for design and to improve the expertise of the designer by providing a theoretical foundation based on logical, rational thought processes and tools. Axiomatic design theory is based on two fundamental axioms that minimize the possibility of making mistakes when products are being developed. The theory helps to overcome shortcomings of the product development process based on a recursive "design/build/test" cycle, which requires continuing modifications and changes as design flaws are discovered through the testing [11].

Axiomatic design consists of four domains and two axioms. The four domains, ordered in consideration, are: customer domain, functional domain, physical domain and process domain. An earlier domain in the series represents "what we want to achieve", whereas the subsequent adjacent domain represents the design solution of "how we propose to satisfy the requirements specified in the prior domain." The customer domain is characterized by customer attributes (CAs), or what the customer is looking for in a product, process, systems or materials. In the functional domain, the customer's needs are specified in terms of functional requirements (FRs) and constraints. To satisfy the specified FR, design parameters (DPs) in the physical domain can be conceived. Finally, to produce the product specified in terms of DPs, a process that is characterized by process variables (PVs) in the process domain can be developed. Axiomatic design activity consists of mapping from one domain to the other, and domains have different meanings in different research areas.

The mapping process between the domains can be expressed mathematically in terms of characteristic vectors that define the design goals and design solutions. At a given level of the design hierarchy, the set of functional requirements that define the specific design goals constitutes the FR vector in the functional domain. Similarly, the set of design parameters in the physical domain that have been chosen to satisfy the FRs constitutes the DP vectors. The mathematical expression can be written as $[\mathrm{FR}]=[\mathrm{A}][\mathrm{DP}]$, where $[\mathrm{A}]$ is called the design matrix that characterizes the product design. The design matrix is of the following form for a design that has three FRs and three DPs, This equation can be written in terms of its elements as equation 1 or 2:

$$
\begin{gathered}
F R_{1}=A_{11} D P_{1}+A_{12} D P_{2}+A_{13} D P_{3} \\
F R_{2}=A_{21} D P_{1}+A_{22} D P_{2}+A_{23} D P_{3} \\
F R_{3}=A_{31} D P_{1}+A_{32} D P_{2}+A_{33} D P_{3} \\
{[\mathrm{FR}]=[\mathrm{A}][\mathrm{DP}]} \\
{[A]=\left[\begin{array}{lll}
A_{11} & A_{12} & A_{13} \\
A_{21} & A_{22} & A_{23} \\
A_{31} & A_{32} & A_{33}
\end{array}\right]}
\end{gathered}
$$


where the two axioms in axiomatic design are identified by examining the common elements that are always present in good designs. The axioms are also identified by examining actions taken during the design stage that result in dramatic improvements.

The first axiom, called the Independence Axiom, states that the independence of FRs must always be maintained, where FRs are defined as the minimum set of independence requirements that characterizes the design goals. The Independence Axiom states that when there are two or more FRs, the design solution must be such that each one can be satisfied without affecting other FRs. That means that a correct set of DPs must be chosen to be able to satisfy FRs and maintain their independence.

The second axiom suggests that physical integration is desirable to reduce the information content if the functional independence can be maintained. This axiom is called the Information Axiom, and it states that among those designs that satisfy the Independence Axiom, the design that has the smallest information content is the best. To make the design work, the information amount must be estimated by the users. Since the information content is defined in terms of probability, the second axiom also states that the design that has the highest probability of success is the best design.

During the mapping process (e.g., from functional domain to physical domain), one must first make the correct design decisions using the Independence Axiom. When several designs that satisfy the Independence Axiom are available, the Information Axiom can be applied to select the best design. To satisfy the Independence Axiom, the design matrix must be either diagonal or triangular. When the design matrix is diagonal (e.g. [A] in equation 4), each of the FRs can be satisfied independently by means of one DP. Such a design is called an uncoupled design. When the matrix is triangular (e.g. [B] in equation 5), the independence of FRs can be guaranteed if and only if the DPs are determined in a proper sequence. Such a design is called a decoupled design. Any other form of the design matrix is called a full matrix and results in a coupled design, for which the end result will be conflicting control. Therefore, when several FRs must be satisfied, designs must be developed that will enable creation of a diagonal or triangular design matrix:

$$
\begin{aligned}
& {[A]=\left[\begin{array}{ccc}
A_{11} & 0 & 0 \\
0 & A_{22} & 0 \\
0 & 0 & A_{33}
\end{array}\right]} \\
& {[B]=\left[\begin{array}{ccc}
B_{11} & 0 & 0 \\
B_{21} & B_{22} & 0 \\
B_{31} & B_{32} & B_{33}
\end{array}\right]}
\end{aligned}
$$

In a design problem, there can be many designs (different sets of FRs) that satisfy the Independence Axiom. However, one of those designs is likely to be superior. The Information Axiom provides a quantitative measure of the merits of a given design and is useful in selecting the best among the acceptable designs. In addition, the Information Axiom provides the theoretical basis for design optimization and robust design, stating that the design with minimum information content is the best. 
According to this axiom, the design with the smallest $I$ is best because it requires the least amount of information to achieve the design goal. Information content $I_{i}$ for a given $\mathrm{FR}_{\mathrm{i}}$ is defined in terms of the probability $P_{i}$ of satisfying $\mathrm{FR}_{i}$,

$$
I_{i}=\log _{2} \frac{1}{P_{i}}=-\log _{2} P_{i}
$$

The information content is expressed in bits of information. When all $m$ FRs are statistically independent, the information content for the system is,

$$
I_{s y s}=\sum_{i=1}^{m} I_{i}=-\sum_{i=1}^{m} \log _{2} P_{i}
$$

When all $m$ FRs are not statistically independent, the information content for the system (shown in equation 7) becomes,

$$
I_{s y s}=\sum_{i=1}^{m} I_{i}=-\sum_{i=1}^{m} \log _{2} P_{i\lfloor j\}} \quad \text { for }\{\mathrm{j}\}=\{1, \ldots, \mathrm{i}-1\}
$$

Applications. Axiomatic design has been applied to a variety of products and systems such as mechanical design, system design and control, software design, organizations management, materials design and more. Many innovations have been made and commercialized based on the use of axiomatic design, such as microcellular plastics and woven electrical connectors [11]. Although the meanings of the four domains are quite different from one product area to another, axiomatic design successfully enhances the design quality in different areas. As many case studies presented suggest [11], the performance, robustness, reliability and functionality of products, process, software, systems and organizations are significantly improved when the axioms are satisfied.

Applications to Usability Design. Ergonomic systems must be designed to be robust and efficient in satisfying their FRs and constraints [12]. Unless FRs related to ergonomics are defined at the beginning of the design process, it is difficult to incorporate human factors after the design is completed. It is equally difficult to modify an existing system or product to overcome ergonomic shortcomings. Therefore, it is important to "design it right from the beginning" [12]. Ergonomics has both timeindependent and time-dependent aspects. Some products may not satisfy human factors from the very beginning of product introduction because of deficiencies in design, but even good products may deteriorate as they are being used [12]. To deal with the former, one must be certain that FRs related to human factors are incorporated and satisfied at the time of design.

In recent years, axiomatic design principles have been applied in human factors to ergonomic microscope workstation design [13], biomechanical hand tools [13], and in HCI to information visual design [14] and e-commerce websites [15]. However, there is not yet much research using axiomatic design to improve usability of humancomputer/human-machine interfaces. 


\section{Conceptual Model of Axiomatic Evaluation Method}

Traditional usability evaluation methods are more proper for use on software and websites than on consumer electronic products. Traditional usability evaluation methods may also neglect usability problems related to the complexity of a product, defined here as a measure of uncertainty in achieving the specified FRs. According to research [11], one type of complexity named imaginary complexity occurs because users lack the knowledge about the function or design. Complexity will cause more errors and require a longer time for users to operate a function. Therefore, by using the "axiomatic evaluation" method developed from the axiomatic design theory, it is possible to discover usability problems regarding complexity of design.

Axiomatic design is different from traditional design theories because it starts designing from "what we want to achieve" to "how we could achieve it", with the former listed as domain vectors and is the latter examined through the mapping matrix between two domains. The complete design process starts from "customer domain" to "functional domain", to "physical domain", to "process domain". Since the final goal of usability evaluation is to examine and enhance the design's efficiency, as well as effectiveness and customer satisfaction, it is meaningful and feasible to start the "mapping" process from "what do customers want" and proceed to "what does the product provide". The framework of axiomatic design is used to set up three domains: "Customer Domain", "Functional Domain" and "Control Domain" and the mappings between them. Table 1 compares the domains, mappings, axioms, and constraints for the axiomatic design and axiomatic evaluation method. The biggest difference in domain settings is that "Control Domain" is the $3^{\text {rd }}$ domain instead of "Physical Domain". The main reason for this change is that as an evaluation process, the physical design variables or parameters cannot be obtained. What can be obtained easily are the control parameters, which are the control keys. The "Customer Domain" [CA] can be retrieved by an open-ended questionnaire. Participants can be provided a list of existing functions and asked what their expected functions are, or any extra expectations. The "Function Domain" [FR] and "Control Domain" [CT] can be gained by interviewing the designer as well as reading the product manual. The uses of the two axioms are similar, but the axiomatic evaluation method has extended constraints for information appliance design such as stimulus-response compatibility [16] and Hick's Law [17], according to which response time increases as a function of the amount of uncertainty among alternatives, or, the amount of information conveyed by an action.

Usability problems can be found by examining the mapping between the three domains. The mapping can be expressed as matrices $[\mathrm{X}]$ and $[\mathrm{Y}]$ :

[Customer Requirement $]=[\mathrm{X}][$ Function $]$

$$
[\text { Function }]=[\mathrm{Y}][\text { Control }]
$$

If the $[\mathrm{X}]$ matrix has a " 0 " row, it means that the customer requirement could not be met, and the function needs to be added to that product if several participants have the same response. If the $[\mathrm{X}]$ matrix has a " 0 " column, it means that there are redundant functions, and we need to delete the function if all or most participants show the "0" column. 
Table 1. Comparison of Axiomatic Design Theory and Axiomatic Evaluation Method

\begin{tabular}{|c|c|c|}
\hline & Axiomatic Design Theory & Axiomatic Evaluation Method \\
\hline $1^{\text {st }}$ domain & $\begin{array}{l}{[\mathrm{CA}] \text { : Customer domain. }} \\
\text { The needs (or attributes) that the } \\
\text { customer is looking for in a } \\
\text { product or process or systems or } \\
\text { materials. }\end{array}$ & $\begin{array}{l}\text { [CA]: Customer domain. } \\
\text { Customer requirements of a } \\
\text { certain product. }\end{array}$ \\
\hline $2^{\text {nd }}$ domain & $\begin{array}{l}{[\mathrm{FR}] \text { : Functional domain. }} \\
\text { The minimum set of independent } \\
\text { requirements that completely } \\
\text { characterizes the functional needs } \\
\text { of the product }\end{array}$ & $\begin{array}{l}\text { [FR]: Functional domain. } \\
\text { The existing functions of a } \\
\text { certain product. }\end{array}$ \\
\hline $3^{\text {rd }}$ domain & $\begin{array}{l}\text { [DP]: Physical domain. } \\
\text { The key physical variables in the } \\
\text { physical domain that characterize } \\
\text { the design that satisfies the } \\
\text { specified FRs. }\end{array}$ & $\begin{array}{l}\text { [CT]: Control domain. } \\
\text { The control keys or elements of } \\
\text { the product. }\end{array}$ \\
\hline $4^{\text {th }}$ domain & $\begin{array}{l}{[\mathrm{PV}] \text { : Processing domain. }} \\
\text { The key variables in the process } \\
\text { domain that characterize the } \\
\text { process that can generate the } \\
\text { specified DPs. }\end{array}$ & Not defined. \\
\hline $\begin{array}{l}\text { Independent } \\
\text { Axiom }\end{array}$ & $\begin{array}{l}\text { The independence of functional } \\
\text { requirement (FRs) must always } \\
\text { be maintained. The design } \\
\text { solution must be such that each } \\
\text { one of the FRs can be satisfied } \\
\text { without affecting other FRs. That } \\
\text { means we have to choose a } \\
\text { correct set of DPs to be able to } \\
\text { satisfy the FRs and maintain their } \\
\text { independence. }\end{array}$ & $\begin{array}{l}\text { The independence of functions } \\
\text { must be maintained. Each } \\
\text { function must be able to be } \\
\text { executed by control keys } \\
\text { without conflicting. Moreover, } \\
\text { control keys for the same } \\
\text { function should be grouped } \\
\text { together. }\end{array}$ \\
\hline $\begin{array}{l}\text { Information } \\
\text { Axiom }\end{array}$ & $\begin{array}{l}\text { Reduce the information content if } \\
\text { the functional independence can } \\
\text { be maintained. }\end{array}$ & $\begin{array}{l}\text { When the Independent Axiom } \\
\text { is satisfied, this axiom is used } \\
\text { to reduce the information } \\
\text { amount, and reduce choices in } \\
\text { decision making for users so } \\
\text { that they could decrease } \\
\text { reaction time and reduce error } \\
\text { rate. }\end{array}$ \\
\hline Constraints & $\begin{array}{l}\text { No constraints for the general } \\
\text { Axiomatic Design method. } \\
\text { There are different constraints } \\
\text { applied in various areas }\end{array}$ & $\begin{array}{l}\text { Decision Making } \\
\text { Hick-Hyman Law } \\
\text { Stimulus-Response } \\
\text { Compatibility }\end{array}$ \\
\hline
\end{tabular}


The mapping between [CA] and [FR] also provides an index of function sufficiency which can be used at the beginning stage of product development. If the average index is high, the current product could be said to satisfy most customers. Otherwise, the product needs to be added more functions.

The mapping between the Functional Domain and the Control Domain shows how easy it is to control the appliance. According to the "Independence Axiom", an ideal matrix should be an uncoupled matrix, or at least a decoupled matrix. An uncoupled matrix is a diagonal matrix, which means the users only need one control step, or one button to operate the function. However, most consumer electronic products have more than 20 functions, and it is difficult to have more than 20 buttons on the panel. Thus, several keys usually need to be pressed to accomplish a certain task.

A decoupled matrix is another option. This means that customers may need to press several keys to achieve their goal, but the keys should not conflict with other functions. Moreover, if the Information Axiom is satisfied, usability constraints for the control keys should also be met. For instance, according to action selection studies, keys for the same task should be grouped together. Therefore, by examining [Y], one can find out whether the Independence Axiom and usability constraints are satisfied.

$$
\left[\begin{array}{c}
\text { Make phone calls } \\
\text { View recent calls } \\
\vdots
\end{array}\right]=\left[\begin{array}{ccc}
Y & \cdots & 0 \\
\vdots & \ddots & \vdots \\
Y & \cdots & Y
\end{array}\right]\left[\begin{array}{c}
\text { Key } 1 \text { (green call key) } \\
\text { Key 2 (red stop key) } \\
\vdots \\
\text { key n }
\end{array}\right]
$$

Usability problems can be found by recording the participants' operating action. If a participant's requirement for the cell phone is to send messages, s/he will be asked to finish the task of sending a message while their operating action is recorded and arranged to the matrix [Y]. If the [Y] matrix is a decoupled matrix, then it means there are no conflicting controls between different functions. If it is not a decoupled matrix, the designers should redesign the controls. However, only meeting the aim of a decoupled matrix is not enough. The designer needs to make sure that users do not need to make too many key presses, and for the same function the keys should be grouped together.

The procedure of the axiomatic evaluation method should be used during the first stage of development. Potential advantages of using the new evaluation method are:

a) Only a few experts are needed. One or two experts knowledgeable about usability and axiomatic evaluation would be enough.

b) A large number of participants may not be needed. Choosing participants that represent the composition of the customers would be sufficient.

c) Extra problems can be discovered. The current usability evaluation method of Heuristic Evaluation is based on the "guidelines", and Cognitive Walkthrough is based on "a designed task". These usability evaluation methods might lead to usability interface problems but neglect other types of usability problems between the interface and other parts of the device such as the control panel. Axiomatic evaluation is more related to customers and would discover the problems that are of concern to them. Examining the mapping between [CA] and [FR] would show what the missing content is (that customers need) and what the redundant content 
is (that is barely needed by customers). By examining the mapping between [FR] and $[\mathrm{CT}]$, one would know what the problems are between the content/function and operation of the content/function.

d) Direct solutions for the discovered usability problems related to controlling can be given. One solution is to rearrange the use of keys or buttons and make the [Y] as a less coupled design to fulfill the Independence Axiom. Another solution is to reduce the number of choices to fulfill the Information Axiom. Other possible solutions include rearranging the keys on the panel so that the stimulus-response compatibility principles apply.

\section{Follow-Up Study and Conclusions}

Based on this conceptual model, an experiment was designed to test the axiomatic evaluation method [18]. Sixty people were randomly assigned to a group using a think aloud method and a group using used the axiomatic evaluation method. Three popular consumer electronic devices representing different levels of complexity were tested by each participant. More usability problems with the cell phone were found by the axiomatic evaluation method than by the other methods. Across all three products, the axiomatic evaluation method was better in finding problems of user requirements. These results suggest that traditional usability methods are not sufficient for evaluating complex devices like smart phone and GPS. Instead, the axiomatic method should be used.

\section{References}

1. Han, S.H., Yun, M.H., Kim, K.-J., Kwahk, J.: Evaluation of Product Usability: Development and Validation of Usability Dimensions and Design Elements Based on Empirical Models. International Journal of Industrial Ergonomics 26, 477-488 (2000)

2. Zhang, T., Rau, P.-L.P., Salvendy, G.: Exploring Critical Usability Factors for Handsets. Behaviour \& Information Technology 29(1), 45-55 (2010)

3. Venkatesh, V., Ramesh, V., Massey, A.P.: Understanding Usability in Mobile Commerce. Communications of the ACM 46(12), 53-56 (2003)

4. Kim, J., Han, S.H.: A Methodology for Developing a Usability Index of Consumer Electronic Products. International Journal of Industrial Ergonomics 38, 333-345 (2008)

5. Ciavarella, C., Paterno, F.: Design Criteria for Location-aware, Indoor, PDA Applications. In: Chittaro, L. (ed.) Mobile HCI 2003. LNCS, vol. 2795, pp. 131-144. Springer, Heidelberg (2003)

6. Ji, Y.G., Park, J.H., Lee, C., Yun, M.H.: A Usability Checklist for the Usability Evaluation of Mobile Phone User Interface. International Journal of Human-Computer Interaction 20(3), 207-231 (2006)

7. Ho, S.Y., Kwok, S.H.: The Attraction of Personalized Service for Users in Mobile Commerce: an Empirical Study. ACM SIGecom Exchanges 3, 10-18 (2002)

8. Bruner, G.C., Kumar, A.: Explaining Consumer Acceptance of Handheld Internet Devices. Journal of Business Research 58, 553-558 (2005)

9. Suh, N.P.: The principles of design. Oxford University Press, New York (1990) 
10. Lee, D.G., Suh, N.: Axiomatic Design and Fabrication of Composite Structures: Applications in Robots, Machine Tools, and Automobiles. Oxford series on advanced manufacturing, p. 451. Oxford University Press, US (2006)

11. Suh, N.P.: Axiomatic design: advances and applications. Oxford University Press, New York (2001)

12. Suh, N.P.: Ergonomics, Axiomatic Design and Complexity Theory. Theoretical Issues in Ergonomics Science 8(2), 101-121 (2007)

13. Helander, M., Lin, L.: Axiomatic Approach in Ergonomics Design with an Extension of the Information Axiom. Journal of Engineering Design 13(4), 321-339 (2002)

14. Quill, L.L., Batchelor, C., Kancler, D.E., Revels, A.R.: Application of Information Visualization Principles at Various Stages of System Development. In: Proceedings of 45th Human Factors and Ergonomics Annual Meeting, pp. 1713-1717 (2001)

15. Yenisey, M.M.: Axiomatic Design Approach for E-commercial Web Sites. LNCS, pp. 308-315. Springer, Heidelberg (2007)

16. Proctor, R.W., Vu, K.-P.L.: Selection and Control of Action. In: Salvendy, G. (ed.) Handbook of Human Factors and Ergonomics, 3rd edn., pp. 89-110. John Wiley, Hoboken (2006)

17. Schneider, D.W., Anderson, J.R.: A Memory-based Model of Hick's Law. Cognitive Psychology 62, 193-222 (2011)

18. Guo, Y., Zhu, Y., Salvendy, G., Proctor, R.W.: Axiomatic Usability Evaluation Method for Complex Information Appliances (under review) 